\title{
Recent Advances in Acousto-Optic Instrumentation for Astronomy
}

\author{
K.B. YushKov ${ }^{a}$, S.P. Anikin ${ }^{a}$, S.I. Chizhikov ${ }^{a}$, V.F. Esipov ${ }^{b}$, A.I. Kolesnikov ${ }^{c}$, \\ O.Yu. MAKAROV ${ }^{a}$, V.YA. MOLChAnOV ${ }^{a}$, S.A. POTANin ${ }^{b}$ And A.M. TATARNIKOV ${ }^{b}$ \\ ${ }^{a}$ Acousto-optical Research Center, National University of Science and Technology "MISIS", \\ 4 Leninsky prospekt, 119049 Moscow, Russia \\ ${ }^{b}$ Sternberg Astronomical Institute, Lomonosov Moscow State University, \\ 13 Universitetsky prospekt, 119991 Moscow, Russia \\ ${ }^{c}$ Tver State University, 33 Zhelyabova str., 170100 Tver, Russia
}

\begin{abstract}
We report recent progress and new developments of acousto-optic imaging spectrometers and spectropolarimeters for astronomical observations. Prototype performance of hyperspectral visible-to-near-UV and visible-to-nearIR imaging systems is demonstrated. Wide-angle acousto-optic paratellurite monochromators were designed and fabricated. The problem of artifacts in spectral images of celestial objects is studied.
\end{abstract}

DOI: $10.12693 /$ APhysPolA.127.81

PACS: 07.60.Rd, 42.30.Va, 42.79.Jq

\section{Introduction}

Since the invention of acousto-optical tunable filters (AOTFs), numerous applications of them in astronomy were reported [1-5]. The construction of most of the imaging spectrometers was based on noncollinear geometry of acousto-optic diffraction with non-critical phase matching, however the design of AOTFs and optical systems as well was improved with time. The progress in AOTF instrumentation resulted in the improvement of filtered image quality. Modern AOTF-based imaging spectrometers combine high spectral resolution with high spatial resolution. For example, in Fig. 1 recognition of Jupiter and its moon Io is demonstrated by means of spectral imaging in visible and NIR. The results were obtained in September 2011 using an AOTF-based spectrometer with the spatial resolution of 1 arcsec and the spectral passband $10 \AA$ at the central wavelength $6563 \AA\left(H_{\alpha}\right.$ emission line). The paratellurite-based monochromator with the clear aperture $15 \times 15 \mathrm{~mm}^{2}$ was designed and fabricated at the Acousto-optical Research Center of the NUST "MISIS", the observations were performed with the Zeiss-600 telescope at the Crimean station of Lomonosov Moscow State University.

In this paper, we report new trends in the design of imaging spectrometers for astronomy and the results of astronomical observations obtained with AOTF-based imaging spectrometers. Three different concepts of imaging spectrometers are compared from the point of view of imaging artifacts.

\section{Different concepts of AOTF design}

It is well known that non-collinear AOTFs suffer from lateral chromatic aberration that is intrinsic to anisotropic Bragg diffraction [6]. A solution to this problem was found as a tilt angle of the output facet of the

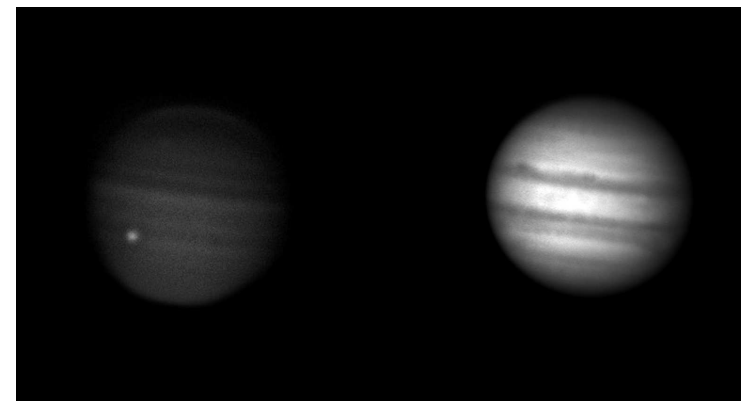

Fig. 1. Spectral images of Jupiter and its moon Io obtained with an acousto-optical spectrometer: left at the wavelength $891 \mathrm{~nm}$ (methane absorption band), right at the wavelength $630 \mathrm{~nm}$ (in the continuum). Visible diameter of Jupiter is $\approx 30$ arcsec, visible diameter of Io is $\approx 1$ arcsec.

crystal, as shown in Fig. 2a, input polarization of light is assumed to be ordinary. There is also an intermediate configuration of the AOTF, when partial compensation of the image shift takes place, and the diffracted beam is parallel to the incident one [5]. This compensation of image shift can be performed for one output polarization only, either for ordinary or for extraordinary. For the orthogonal polarization, the image shift increases. For that reason, AOTFs for spectropolarimetric imaging are usually designed with parallel optical facets [4, 7], as shown in Fig. 2b. As an alternative to these two AOTF designs, we propose an AOTF with enhanced angular dispersion. Thus a greater spatial separation of diffraction by the transmission function side-lobes can be obtained (Fig. 2c).

Precise control of ghosts, i.e. unwanted transmitted spectral components of input emission, is a key to obtaining reliable observation results with spectral instruments. 


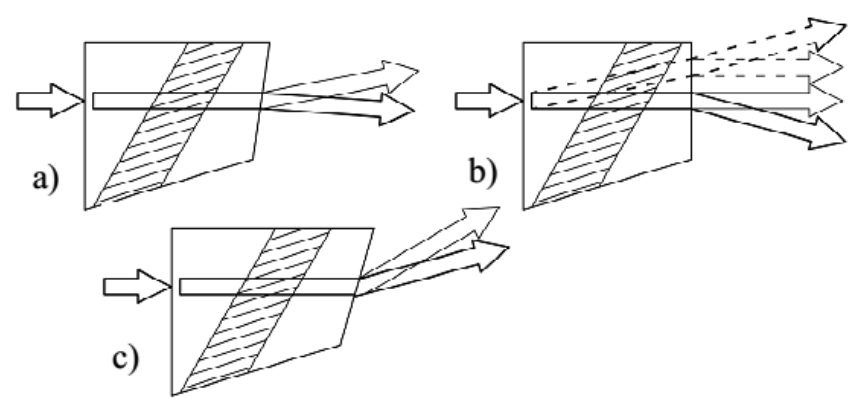

Fig. 2. Different configurations of non-collinear imaging AOTFs: (a) output wedge cut for minimization of image scanning, (b) plane parallel face cut for spectropolarimetric imaging observations, (c) output wedge cut for increasing angular dispersion.

When we consider hyperspectral imaging, the ghosts can be classified as follows:

1) If an imaging spectrometer is designed with maximum compensation for angular dispersion, all the spectral components that are transmitted by the filter coincide at the detector array. In that case, degradation of spatial resolution does not take place, however the measured data can be incorrect. The observed intensity distribution is a sum of the actual distribution of light corresponding to the central wavelength and out-of-bandwidth transmitted light.

2) For a spectral imager with low angular dispersion, partial overlapping of actual image with the ghosts diffracted by the sidelobes of AOTF transmission function can take place. As a result, the quality of obtained images noticeably degrades. This phenomenon is known in acousto-optical hyperspectral imaging systems [8, 9].

3) A spectral imager with high angular dispersion can be effective for eliminating ghost images in observations of the objects with discrete emission spectra. The necessary amount of angular dispersion must be correlated with the size of the observed object and its spectrum: the separation of images obtained in the neighboring emission lines must be greater than the size of the object.

Demonstration of the influence of angular dispersion on the performance of the AOTF-based spectrometer is provided in Fig. 3 using a stellar image. The intensity dis-
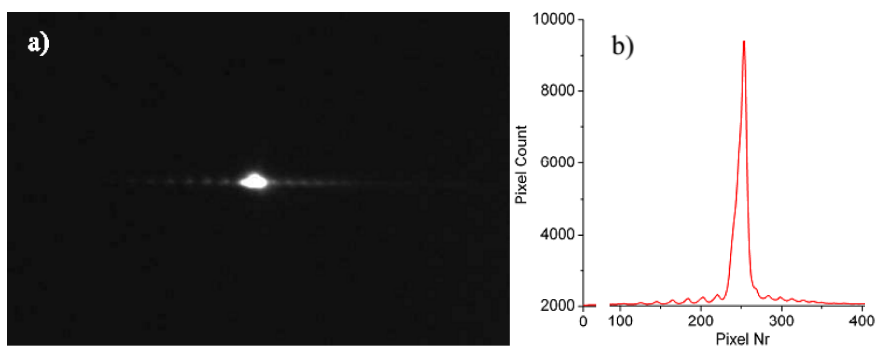

Fig. 3. Diffracted spectral image of the star Vega $(\alpha-$ Lyr) at the wavelength $5320 \AA$ (a) and correspondent horizontal intensity profile (b). tribution in the image is the point-spread function (PSF) of the imaging system including atmospheric turbulence, pupil diffraction, and angular dispersion of the AOTF. Numerous side-lobes of the PSF are visible because the spectrum of the star is continuous, with narrow absorption lines.

\section{Spectral artifacts in acousto-optical imaging}

Among celestial objects, different types of emission spectra are observed. Stars have continuous black-body spectra with either absorption or emission lines. Planets and other Solar system objects reflect solar light and their spectrum contains footprints of surface reflection and atmospheric absorption. Planetary nebulae have emission spectra consisting of discrete lines. Spectral imaging of nebulae is a powerful tool for studying their morphology as well as it enables mapping of chemical elements in extended objects.

For objects with discrete emission spectra any emission line can produce a ghost image. The situation can be especially dramatic when a weak line is observed at the presence of a strong neighboring emission line. That usually takes place when those emission lines belong to different chemical elements.
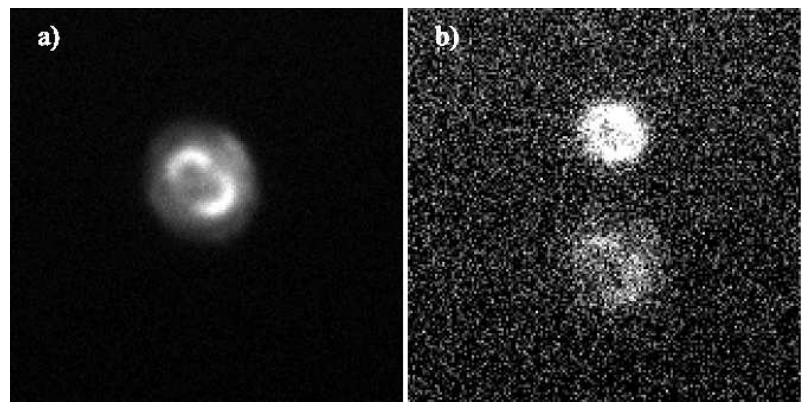

Fig. 4. Spectral images of NGC7662 planetary nebula obtained with an acousto-optical spectrometer: (a) forbidden [OIII] line $5007 \AA$, (b) HeII line $4686 \AA$ (top) and the phantom image in [OIII] line $5007 \AA$ (bottom) transmitted by the spectral side-lobe of the transmission function.

As an object for observations we have chosen NGC 7662 "Blue snowball" nebula [10]. In Fig. 4 the spectral images of obtained with an AOTF-based spectrometer are shown. Observations were made in October 2012 using an AOTF-based spectrometer with the monochromator designed with the zero tilt angle of the output facet, as shown in Fig. 2b. The AOTF with clear aperture $23 \times 23 \mathrm{~mm}^{2}$ had the passband of $4.9 \AA$ at the central wavelength $4880 \AA$ (Ar-laser emission line). At the output of the AOTF, only the extraordinary diffracted beam was imaged onto the CCD.

The NGC 7662 nebula has the intensity of the [OIII] emission line at $5007 \AA$ almost 20 times higher than the intensity of the HeII emission line at $4686 \AA$. As a result, long exposure image at $4686 \AA$ line contains the 
ghost image of the [OIII] emission line. Comparison of nebula spectral images obtained with the AOTF at different emission lines demonstrates that ghost images caused by diffraction of light by the sidelobes of the PSF can be an issue during observation of extended objects if angular dispersion is not specially considered for the diffracted beams.

\section{Aberration issues}

Another problem in obtaining diffraction-limited quality of images at the output of AOTFs consists in different aberrations. Longitudinal chromatic aberrations are caused by the dispersion of refractive indices of acoustooptic crystals [9]. Without special care of the problem, that type of aberrations results in defocusing of the image while tuning the transmitted optical wavelength [11]. Accurate analysis of interaction geometry in AOTFs shows that image distortions take place during anisotropic Bragg diffraction [12]. In this paper, our attention is focused on a different type of aberrations in AOTF-based imaging spectrometers that was not studied before, namely on astigmatism. The problem of correction for astigmatism was addressed briefly in Ref. [1] for collinear AOTFs only.

In noncollinear AOTFs with non-critical phase matching, the diffracted beam in the crystal changes its direction. The inclination of the beam can be compensated with a special tilt angle of the output crystal facet; however in general, the diffracted beam at the output of the AOTF changes its direction. As a result, axial symmetry of the optical system is violated, and the diffraction plane becomes a special direction for the optical system of the imaging spectrometer. Thus, from the point of view of geometrical optics, an AOTF can be approximated as a prism, which inevitably causes astigmatism. Compensation for that astigmatism in such systems can be made using passive optical elements, e.g. prisms, tilted plates, or cylindrical lenses.

\section{Summary}

In the paper, we observed the artifacts in conventional acousto-optical imaging spectrometers and proposed a new concept of acousto-optic imaging spectrograph for observation of astronomical objects with discrete spectra. The prism at the output of the AOTF increases the angular dispersion of the Bragg diffraction. Thus it is possible to separate the ghost spectral images.

\section{Acknowledgments}

The work was carried out with financial support in part from the Ministry of Education and Science of the Russian Federation in the framework of Increase Competitiveness Program of NUST "MISiS" (No. K1-2014-008) and from the Russian Foundation for Basic Research in project No. 12-07-00640.

\section{References}

[1] R.B. Wattson, S.A. Rappaport, E.E. Frederick, Icarus 27, 417 (1976).

[2] B. Bates, D. Halliwell, D. Findlay, Appl. Opt. 23, 257 (1994).

[3] W. Hayden Smith, K.M. Smith, Exp. Astron. 1, 329 (1990).

[4] D.A. Glenar, J.J. Hillman, B. Saif, J. Bergstrahl, Appl. Opt. 33, 7412 (1994).

[5] V.Ya. Molchanov, V.M. Lyuty, V.F. Esipov, S.P. Anikin, O.Yu. Makarov, N.P. Solodovnikov, Astron. Lett. 28, 713 (2002).

[6] T. Yano, A. Watanabe, Appl. Opt. 15, 2250 (1976).

[7] V.Ya. Molchanov, S.P. Anikin, S.I. Chizhikov, K.B. Yushkov, O.Yu. Makarov, A.M. Tatarnikov, S.A. Potanin, V.F. Esipov, Proc. SPIE 9147, 91472T (2014).

[8] V.B. Voloshinov, D.V. Bogomolov, Quant. Electron. 36, 457 (2006)

[9] V.B. Voloshinov, K.B. Yushkov, B. Linde, J. Opt. A Pure Appl. Opt. 9, 341 (2007).

[10] S. Hyung, L.H. Adler, Astrophys. J. 491, 242 (1997).

[11] V.B. Voloshinov, K.B. Yushkov, T.V. Yukhnevich, Moscow Univ. Phys. Bull. 67, 437 (2012).

[12] V. Pozhar, A. Machihin, Appl. Opt. 51, 4513 (2014). 